\title{
Commuting functions and simultaneous Abel equations
}

\author{
by W. JARCZYK (Katowice), K. Łoskot (Katowice) and \\ M. C. ZDUN (Kraków)
}

Abstract. The system of Abel equations

$$
\alpha\left(f_{t}(x)\right)=\alpha(x)+\lambda(t), \quad t \in T,
$$

is studied under the general assumption that $f_{t}$ are pairwise commuting homeomorphisms of a real interval and have no fixed points ( $T$ is an arbitrary non-empty set). A result concerning embeddability of rational iteration groups in continuous groups is proved as a simple consequence of the obtained theorems.

Introduction. We study continuous solutions of the system of Abel equations

$$
\alpha\left(f_{t}(x)\right)=\alpha(x)+\lambda(t), \quad t \in T,
$$

where $T$ is a non-empty set, $\lambda: T \rightarrow \mathbb{R}$ is a given function and $f_{t}, t \in T$, are pairwise commuting homeomorphisms of an interval $(a, b)(-\infty \leq a<$ $b \leq \infty)$. We assume that the homeomorphisms $f_{t}$ have no fixed points and satisfy some additional conditions (hypotheses $\left(\mathrm{H}_{1}\right)-\left(\mathrm{H}_{3}\right)$ below). Under these assumptions we describe solutions of system (1) by examining the orbits of the group generated by $\left\{f_{t}: t \in T\right\}$. Our results generalize some of those obtained by the third author in [4] and [5].

In Section 1 we formulate the basic definitions and main results describing the iteration properties of $\left\{f_{t}: t \in T\right\}$ (Propositions 1 and 2) and continuous solutions of system (1) (Theorem 1). Section 2 contains the proofs of these results and a number of auxiliary lemmas. There we also prove a slight generalization of the Krylov-Bogolyubov theorem (Theorem 2) which serves as the main tool in the proof of Theorem 1. The last part of the paper provides some consequences of Theorem 1 concerning system (1) (Theorems 3

1991 Mathematics Subject Classification: Primary 39B12; Secondary 26A18, 58F08.

Key words and phrases: Abel equation, commuting functions, iteration group. 
and 4, Corollaries 3 and 4) as well as the problem of embeddability of rational iteration groups in continuous groups (Corollary 2).

1. Basic notions and main results. Let $(a, b)(-\infty \leq a<b \leq \infty)$ be an interval. In what follows $\mathcal{A}$ will stand for the set of all homeomorphisms mapping $(a, b)$ onto itself and having no fixed points. Observe that any element of $\mathcal{A}$ is a strictly increasing function.

The following notions play a fundamental role in the paper.

Given commuting homeomorphisms $f$ and $g$ mapping $(a, b)$ onto itself we say that the pair $(f, g)$ is periodic if

$$
\forall_{x \in(a, b)} \exists_{m, n \in \mathbb{Z} \backslash\{0\}} f^{m}(x)=g^{n}(x),
$$

and $(f, g)$ is aperiodic if

$$
\forall_{x \in(a, b)} \forall_{m, n \in \mathbb{Z} \backslash\{0\}} f^{m}(x) \neq g^{n}(x) .
$$

(Here and in what follows the symbol $f^{m}$ stands for the $m$ th iterate of $f$. Alternatively, periodic and aperiodic pairs are called iteratively commensurable and iteratively incommensurable, respectively.)

Re mark 1 ([2, Corollary 4.2]). Let $f, g \in \mathcal{A}$ commute. The pair $(f, g)$ is periodic if and only if $f^{m}=g^{n}$ for some non-zero integers $m, n$.

Let $f, g \in \mathcal{A}$ commute. For every $x \in(a, b)$ there exists a (unique) sequence $\left(s_{k}(x): k \in \mathbb{N}\right)$ of integers such that

$$
f^{s_{k}(x)+1}(x) \leq g^{k}(x)<f^{s_{k}(x)}(x), \quad k \in \mathbb{N},
$$

provided $f<\mathrm{Id}$, and

$$
f^{s_{k}(x)+1}(x) \geq g^{k}(x)>f^{s_{k}(x)}(x), \quad k \in \mathbb{N},
$$

if $f>$ Id. It follows from [7, Theorem 1, Remark 2 and Lemma 4] that for every $x \in(a, b)$ there exists a finite non-zero limit

$$
\nu(f, g)=\lim _{k \rightarrow \infty} s_{k}(x) / k
$$

which actually does not depend on $x$.

Remark 2. Let $f, g \in \mathcal{A}$ commute.

(i) $([7$, Corollary 1 and Remark 2]) $\nu(f, g)$ is irrational if and only if $(f, g)$ is aperiodic.

(ii) $([7$, Theorem 2 and Remark 2]) If $(f, g)$ is periodic and $m, n \in \mathbb{Z} \backslash\{0\}$, then $\nu(f, g)=m / n$ if and only if $f^{m}=g^{n}$.

As follows from the fact below, $\nu(f, g)$ can be defined otherwise (cf. [2, p. 85]).

Remark 3. Let $f, g \in \mathcal{A}$ commute. Then for every $x \in(a, b)$,

$$
\nu(f, g)=\inf \left\{m / n: m \in \mathbb{Z}, n \in \mathbb{N}, f^{m}(x)<g^{n}(x)\right\} \quad \text { if } f<\mathrm{Id},
$$




$$
\nu(f, g)=\sup \left\{m / n: m \in \mathbb{Z}, n \in \mathbb{N}, f^{m}(x)<g^{n}(x)\right\} \quad \text { if } f>\text { Id. }
$$

Proof. By [7, Remark 2 and Lemma 5], $\nu\left(f, g^{-1}\right)=-\nu(f, g)$ and $\nu(g, f)=\nu(f, g)^{-1}$. This allows us to consider only the case where $f<\mathrm{Id}$ and $g<$ Id.

Fix an $x \in(a, b)$ and let $m \in \mathbb{Z}$ and $n \in \mathbb{N}$ be such that $f^{m}(x)<g^{n}(x)$. Observe that necessarily $m \in \mathbb{N}$. Lemma 1 of [7] shows that $f^{m+1}<g^{n}$, whence, by [7, Theorem 3], $m+1>n \nu(f, g)$. Thus

$$
\inf \left\{(m+1) / n: m \in \mathbb{Z}, n \in \mathbb{N}, f^{m}(x)<g^{n}(x)\right\} \geq \nu(f, g) .
$$

On the other hand, if $\left(s_{k}(x): k \in \mathbb{N}\right)$ is a sequence of integers satisfying (2), then

$$
f^{s_{k}(x)+2}(x)<f^{s_{k}(x)+1}(x) \leq g^{k}(x), \quad k \in \mathbb{N},
$$

and $\lim _{k \rightarrow \infty}\left(s_{k}(x)+3\right) / k=\nu(f, g)$ so

$$
\inf \left\{(m+1) / n: m \in \mathbb{Z}, n \in \mathbb{N}, f^{m}(x)<g^{n}(x)\right\} \leq \nu(f, g) .
$$

If $f^{m}(x)<g^{n}(x)$ for $m \in \mathbb{Z}$ and $n \in \mathbb{N}$, then, as one can easily verify, $f^{k m}(x)<g^{k n}(x), k \in \mathbb{N}$. Hence

$$
\begin{aligned}
& \inf \left\{(m+1) / n: m \in \mathbb{Z}, n \in \mathbb{N}, f^{m}(x)<g^{n}(x)\right\} \\
&=\inf \left\{m / n: m \in \mathbb{Z}, n \in \mathbb{N}, f^{m}(x)<g^{n}(x)\right\} .
\end{aligned}
$$

Let $T$ be a non-empty set and consider the following hypothesis.

$\left(\mathrm{H}_{1}\right) f_{t}, t \in T$, are pairwise commuting elements of $\mathcal{A}$ and for every $k \in \mathbb{N}, t_{1}, \ldots, t_{k} \in T$, and $n_{1}, \ldots, n_{k} \in \mathbb{Z}$ either

$$
\forall_{x \in(a, b)} f_{t_{1}}^{n_{1}} \circ \ldots \circ f_{t_{k}}^{n_{k}}(x)=x,
$$

or

$$
\forall_{x \in(a, b)} f_{t_{1}}^{n_{1}} \circ \ldots \circ f_{t_{k}}^{n_{k}}(x) \neq x .
$$

Remark 4. Hypothesis $\left(\mathrm{H}_{1}\right)$ is equivalent to the statement that the group generated by $\left\{f_{t}: t \in T\right\} \subseteq \mathcal{A}$ is commutative and disjoint, that is, Id is its unique element having fixed points.

EXAmple. Let $T$ be a non-empty set of reals not containing zero and let $\alpha$ be a homeomorphism of $(a, b)$ onto $\mathbb{R}$. One can easily check that the functions $f_{t}, t \in T$, given by

$$
f_{t}(x)=\alpha^{-1}(\alpha(x)+t), \quad x \in(a, b),
$$

satisfy $\left(\mathrm{H}_{1}\right)$ and, moreover, $\nu\left(f_{s}, f_{t}\right)=t / s$ for every $s, t \in T$.

Let $\mathcal{F}=\left\{f_{t}: t \in T\right\}$ be a family of pairwise commuting bijections of $(a, b)$. For any $x \in(a, b)$ define the $\mathcal{F}$-orbit of $x$ by

$$
C_{\mathcal{F}}(x)=\left\{f_{t_{1}}^{n_{1}} \circ \ldots \circ f_{t_{k}}^{n_{k}}(x): k \in \mathbb{N}, t_{1}, \ldots, t_{k} \in T, n_{1}, \ldots, n_{k} \in \mathbb{Z}\right\}
$$

and denote by $L_{\mathcal{F}}(x)$ the set of all its cluster points. 
Proposition 1. Let $\mathcal{F}=\left\{f_{t}: t \in T\right\}$ be a family of homeomorphisms satisfying hypothesis $\left(\mathrm{H}_{1}\right)$. Then

(i) $L_{\mathcal{F}}(x)=L_{\mathcal{F}}(y)$ for every $x \in(a, b)$ (from now on we denote this set by $\left.L_{\mathcal{F}}\right)$;

(ii) $f\left(L_{\mathcal{F}} \cap(a, b)\right)=L_{\mathcal{F}} \cap(a, b)$ for every $f \in \mathcal{F}$;

(iii) $L_{\mathcal{F}}$ is either a perfect nowhere dense set containing $\{a, b\}$, or $L_{\mathcal{F}}=$ $\{a, b\}$, or $L_{\mathcal{F}}=[a, b]$.

In what follows $t_{0}$ will be a fixed element of $T$.

Given pairwise commuting elements $f_{t}, t \in T$, of $\mathcal{A}$ we can put

$$
\nu(t)=\nu\left(f_{t_{0}}, f_{t}\right), \quad t \in T .
$$

Observe that $\nu\left(t_{0}\right)=1$ and denote by $G$ the additive group generated by $\nu(T)$. Clearly $G$, being a subgroup of the additive group of reals is either discrete, i.e. $G=p \mathbb{Z}$ for a non-zero $p$, or $G$ is a dense subset of $\mathbb{R}$.

Proposition 2. Let $\mathcal{F}=\left\{f_{t}: t \in T\right\}$ be a family of homeomorphisms satisfying hypothesis $\left(\mathrm{H}_{1}\right)$. Then the following statements are equivalent:

(i) the group $G$ is discrete;

(ii) $L_{\mathcal{F}}=\{a, b\}$;

(iii) there exists a homeomorphism $h \in \mathcal{A}$ and a function $m: T \rightarrow \mathbb{Z}$ such that

$$
f_{t}=h^{m(t)}, \quad t \in T .
$$

ThEOREM 1. Let $\mathcal{F}=\left\{f_{t}: t \in T\right\}$ satisfy hypothesis $\left(\mathrm{H}_{1}\right)$.

(i) Assume that $G$ is discrete. Then the system

$$
\alpha\left(f_{t}(x)\right)=\alpha(x)+\nu(t), \quad t \in T,
$$

has a homeomorphic solution depending on an arbitrary function $\left(^{1}\right)$.

(ii) Assume that $G$ is dense in $\mathbb{R}$. Then (A) has a unique (up to an additive constant) continuous solution $\alpha:(a, b) \rightarrow \mathbb{R}$. This solution is monotonic and $\alpha\left(L_{\mathcal{F}} \cap(a, b)\right)=\mathbb{R}$. Moreover, $\alpha$ is invertible if and only if $L_{\mathcal{F}}=[a, b]$.

In the last section we shall give two versions of Theorem 1 for families satisfying one of the following hypotheses:

$\left(\mathrm{H}_{2}\right) f_{t}, t \in T$, are pairwise commuting elements of $\mathcal{A}$ and $\left(f_{t_{0}}, f_{t}\right)$ is periodic for every $t \in T$.

$\left({ }^{1}\right)$ This means here that there exists an interval $I \subset(a, b)$ such that any continuous function defined on $I$ which is strictly decreasing in the case $f_{t_{0}}<$ Id and strictly increasing provided $f_{t_{0}}>\mathrm{Id}$, can be uniquely extended to a homeomorphic solution $\alpha:(a, b) \rightarrow \mathbb{R}$ of system (A). 
$\left(\mathrm{H}_{3}\right) f_{t}, t \in T$, are elements of $\mathcal{A}$ and there is a $t_{1} \in T$ such that $\left(f_{t_{0}}, f_{t_{1}}\right)$ is aperiodic, and

$$
f_{t_{0}} \circ f_{t}=f_{t} \circ f_{t_{0}} \quad \text { and } \quad f_{t_{1}} \circ f_{t}=f_{t} \circ f_{t_{1}}, \quad t \in T .
$$

We postpone the comparison of hypotheses $\left(\mathrm{H}_{1}\right)-\left(\mathrm{H}_{3}\right)$ to Remark 5 in the next section.

2. Proofs. We start with a version of the Krylov-Bogolyubov theorem, useful in the proof of Theorem 1.

Theorem 2. Let $X$ be a compact metric space and $F_{t}: X \rightarrow X, t \in T$, be pairwise commuting continuous functions. Then there exists a probability Borel measure $\mu$ on $X$ such that

$$
\mu\left(F_{t}^{-1}(A)\right)=\mu(A), \quad t \in T,
$$

for every Borel set $A \subset X$.

P r o o f. Let $\mathcal{C}(X)$ be the space of all continuous real functions on $X$ with the sup norm. According to the Riesz representation theorem the adjoint space $\mathcal{C}(X)^{*}$ can be identified with the space of all countably additive realvalued set functions of bounded variation defined on the $\sigma$-algebra of all Borel subsets of $X$.

For each $t \in T$ the operator $S_{t}: \mathcal{C}(X) \rightarrow \mathcal{C}(X)$ defined by

$$
S_{t} F=F \circ F_{t}
$$

is linear and continuous. Let $P_{t}$ be the adjoint operator to $S_{t}, t \in T$. It can be easily checked that for each $t \in T$,

$$
\left(P_{t} \mu\right)(A)=\mu\left(F_{t}^{-1}(A)\right), \quad \mu \in \mathcal{C}(X)^{*},
$$

and $P_{t}(\mathcal{M}) \subset \mathcal{M}$, where $\mathcal{M}=\left\{\mu \in \mathcal{C}(X)^{*}: \mu \geq 0, \mu(X)=1\right\}$. Moreover, $\mathcal{M}$ is a convex and weak ${ }^{*}$ compact subset of $\mathcal{C}(X)^{*}$ and $P_{t}, t \in T$, are linear and weak* continuous. It is also clear that

$$
P_{s} \circ P_{t}=P_{t} \circ P_{s}, \quad s, t \in T .
$$

Therefore, by the Markov-Kakutani fixed point theorem (see [1, Theorem 2.4]), the operators $P_{t}, t \in T$, have a common fixed point. This completes the proof.

Lemma 1. Assume $\left(\mathrm{H}_{1}\right)$ and let $k \in \mathbb{N}, t_{1}, \ldots, t_{k} \in T, n_{1}, \ldots, n_{k} \in \mathbb{Z}$. If $n_{1} \nu\left(t_{1}\right)+\ldots+n_{k} \nu\left(t_{k}\right)=0$, then $f_{t_{1}}^{n_{1}} \circ \ldots \circ f_{t_{k}}^{n_{k}}=\mathrm{Id}$.

Proof. Notice that, by $\left(\mathrm{H}_{1}\right)$, for every $t \in T$ the pair $\left(f_{t_{0}}, f_{t}\right)$ is either periodic or aperiodic.

Let $n_{1}, \ldots, n_{k}$ satisfy $n_{1} \nu\left(t_{1}\right)+\ldots+n_{k} \nu\left(t_{k}\right)=0$. Without loss of generality we can assume that $f_{t_{0}}(x)>x$ for $x \in(a, b)$. Suppose that $f=f_{t_{1}}^{n_{1}} \circ \ldots \circ f_{t_{k}}^{n_{k}}$ has no fixed point and assume, for instance, that $f(x)>x$ 
for $x \in(a, b)$. Fix an $x_{0} \in(a, b)$ and arbitrary integers $p_{1}, \ldots, p_{k}$ for which $p_{1} \nu\left(t_{1}\right)+\ldots+p_{k} \nu\left(t_{k}\right)>0$. Since

$$
i\left(n_{1} \nu\left(t_{1}\right)+\ldots+n_{k} \nu\left(t_{k}\right)\right)=0<p_{1} \nu\left(t_{1}\right)+\ldots+p_{k} \nu\left(t_{k}\right), \quad i \in \mathbb{N},
$$

it follows from [2, Lemma 4.14] that

$$
f^{i}\left(x_{0}\right)<f_{t_{1}}^{p_{1}} \circ \ldots \circ f_{t_{k}}^{p_{k}}\left(x_{0}\right)<b, \quad i \in \mathbb{N} .
$$

Consequently, $\left(f^{i}\left(x_{0}\right): i \in \mathbb{N}\right)$, being an increasing sequence, has a limit in $(a, b)$, i.e. $f$ has a fixed point contrary to our assumption. Thus, by $\left(\mathrm{H}_{1}\right)$, $f=$ Id.

Given an additive subgroup $H$ of $\mathbb{R}$ and a family $\left\{F_{t}: t \in H\right\} \neq\left\{F_{0}\right\}$ of continuous functions mapping $(a, b)$ onto itself we say that $\left\{F_{t}: t \in H\right\}$ is a disjoint $H$-flow if

$$
F_{s} \circ F_{t}=F_{s+t}, \quad s, t \in H,
$$

and the graphs of any two distinct $F_{t}$ are disjoint.

Lemma 2. Assume $\left(\mathrm{H}_{1}\right)$. Then the formula

$$
\begin{aligned}
F\left(n_{1} \nu\left(t_{1}\right)+\ldots+n_{k} \nu\left(t_{k}\right), \cdot\right)= & f_{t_{1}}^{n_{1}} \circ \ldots \circ f_{t_{k}}^{n_{k}}, \\
& k \in \mathbb{N}, t_{1}, \ldots, t_{k} \in T, n_{1}, \ldots, n_{k} \in \mathbb{Z},
\end{aligned}
$$

correctly defines a disjoint $G$-flow.

Proof. It is enough to observe that, by Lemma 1 , if $k \in \mathbb{N}, t_{1}, \ldots, t_{k} \in T$, $n_{1}, \ldots, n_{k}, m_{1}, \ldots, m_{k} \in \mathbb{Z}$ and $n_{1} \nu\left(t_{1}\right)+\ldots+n_{k} \nu\left(t_{k}\right)=m_{1} \nu\left(t_{1}\right)+\ldots+$ $m_{k} \nu\left(t_{k}\right)$, then $f_{t_{1}}^{n_{1}} \circ \ldots \circ f_{t_{k}}^{n_{k}}=f_{t_{1}}^{m_{1}} \circ \ldots \circ f_{t_{k}}^{m_{k}}$

Proof of Proposition 1. Define a $G$-flow $\left\{F_{t}: t \in G\right\}$ as in Lemma 2. Then $L_{\mathcal{F}}(x), x \in(a, b)$, is just the set of all cluster points of the orbit $\left\{F_{t}(x): t \in G\right\}$. Thus, by [6, Theorem 1], the sets $L_{\mathcal{F}}(x)$ have all the required properties except possibly $a, b \in L_{\mathcal{F}}(x)$. But observe that if $f \in \mathcal{F}$ and, for instance, $f<\mathrm{Id}$, then

$$
\lim _{n \rightarrow \infty} f^{n}(x)=a \text { and } \quad \lim _{n \rightarrow-\infty} f^{n}(x)=b .
$$

LEMMA 3. Let $f_{t}, t \in T$, be pairwise commuting increasing homeomorphisms of $(a, b)$ onto itself and let $n \in \mathbb{Z}$ and $r: T \rightarrow \mathbb{Z}$ be such that

$$
f_{t}^{n}=f_{t_{0}}^{r(t)}, \quad t \in T \text {. }
$$

Then there exists a homeomorphism $h$ of $(a, b)$ onto itself and $m: T \rightarrow \mathbb{Z}$ for which

$$
f_{t}=h^{m(t)}, \quad t \in T
$$


Proof. For each $t \in T$ put $k(t)=\operatorname{gcd}(n, r(t))$. Setting $p(t)=n / k(t)$ and $q(t)=r(t) / k(t)$ for each $t \in T$ we have

$$
\left(f_{t}^{p(t)}\right)^{k(t)}=\left(f_{t_{0}}^{q(t)}\right)^{k(t)}
$$

that is, by Lemma 1 of [4],

$$
f_{t}^{p(t)}=f_{t_{0}}^{q(t)}, \quad t \in T .
$$

Since $\operatorname{gcd}(p(t), q(t))=1$ for $t \in T$, there are $u, v: T \rightarrow \mathbb{Z}$ such that

$$
u(t) p(t)+v(t) q(t)=1, \quad t \in T .
$$

Putting

$$
h_{t}=f_{t_{0}}^{u(t)} \circ f_{t}^{v(t)}, \quad t \in T,
$$

we obtain pairwise commuting increasing homeomorphisms of $(a, b)$ onto itself. For each $t \in T$ we have, by (4) and (5),

$$
h_{t}^{p(t)}=\left(f_{t_{0}}^{u(t)} \circ f_{t}^{v(t)}\right)^{p(t)}=f_{t_{0}}^{u(t) p(t)+v(t) q(t)}=f_{t_{0}}
$$

and

$$
h_{t}^{q(t)}=\left(f_{t_{0}}^{u(t)} \circ f_{t}^{v(t)}\right)^{q(t)}=f_{t}^{u(t) p(t)+v(t) q(t)}=f_{t} .
$$

It follows from the definition of $p$ that the set $p(T)$ is finite. Let $p(T)=$ $p\left(\left\{t_{1}, \ldots, t_{l}\right\}\right)$ with $t_{1}, \ldots, t_{l} \in T$ such that $p\left(t_{i}\right) \neq p\left(t_{j}\right), i, j=1, \ldots, l$, $i \neq j$. Then, by (6) and [4, Lemma 1$]$,

$$
\text { if } p(t)=p\left(t_{i}\right), \quad \text { then } \quad h_{t}=h_{t_{i}}, \quad t \in T, i=1, \ldots, l .
$$

Moreover, by $(6), h_{t_{i}}^{p\left(t_{i}\right)}=h_{t_{j}}^{p\left(t_{j}\right)}, i, j=1, \ldots, l$, and $0 \notin p(T)$. Thus, on account of [2, Lemma 4.9], there exists a homeomorphism $h$ of $(a, b)$ onto itself and integers $w_{1}, \ldots, w_{l}$ such that

$$
h_{t_{i}}=h^{w_{i}}, \quad i=1, \ldots, l .
$$

For each $t \in T$ put $m(t)=w_{i} q(t)$, where $i \in\{1, \ldots, l\}$ is a unique number satisfying $p(t)=p\left(t_{i}\right)$. Then, by $(7)-(9)$,

$$
f_{t}=h_{t}^{q(t)}=h_{t_{i}}^{q(t)}=\left(h^{w_{i}}\right)^{q(t)}=h^{m(t)},
$$

for every $t \in T$, which was to be proved.

Lemma 4. Assume $\left(\mathrm{H}_{2}\right)$. Then the following statements are equivalent:

(i) $G$ is discrete;

(ii) there exists a homeomorphism $h \in \mathcal{A}$ and $m: T \rightarrow \mathbb{Z}$ satisfying (3).

Proof. (i) $\Rightarrow$ (ii). If $G$ is discrete then $\nu(t)=\operatorname{cr}(t), t \in T$, with some $c \in \mathbb{R}$ and $r: T \rightarrow \mathbb{Z}$. Since $\nu\left(t_{0}\right)=1$, we have $c \neq 0$ and, consequently,

$$
r\left(t_{0}\right) \nu(t)=r(t) \nu\left(t_{0}\right), \quad t \in T \text {. }
$$


Thus, by Lemma 4.15 of [2] applied to $f_{t_{0}}, f_{t}$,

$$
f_{t}^{r\left(t_{0}\right)}=f_{t_{0}}^{r(t)}, \quad t \in T .
$$

Therefore, Lemma 3 yields the assertion.

(ii) $\Rightarrow$ (i). Let $h \in \mathcal{A}$ and let $m: T \rightarrow \mathbb{Z}$ satisfy (3). It follows from (3) that $m(t) \neq 0, t \in T$, and

$$
f_{t_{0}}^{m(t)}=h^{m\left(t_{0}\right) m(t)}=f_{t}^{m\left(t_{0}\right)}, \quad t \in T .
$$

Thus, in view of Remark 2(ii), $\nu(t)=\nu\left(f_{t_{0}}, f_{t}\right)=m(t) / m\left(t_{0}\right)$, for each $t \in T$, whence $G=\left(1 / m\left(t_{0}\right)\right) \mathbb{Z}$.

Now we can compare hypotheses $\left(\mathrm{H}_{1}\right)-\left(\mathrm{H}_{3}\right)$.

Remark 5. (i) $\left(\mathrm{H}_{1}\right)$ implies either $\left(\mathrm{H}_{2}\right)$ or $\left(\mathrm{H}_{3}\right)$.

(ii) $\left(\mathrm{H}_{2}\right)$ implies $\left(\mathrm{H}_{1}\right)$.

(iii) If $f_{t}, t \in T$, satisfy $\left(\mathrm{H}_{3}\right)\left({ }^{2}\right)$, then any aperiodic pair $\left(f_{t_{0}}, f_{t}\right)$ satisfies $\left(\mathrm{H}_{1}\right)$.

Proof. Statements (i) and (iii) are obvious. To prove (ii) fix $k \in \mathbb{N}$ and $t_{1}, \ldots, t_{k} \in T$. It follows from $\left(\mathrm{H}_{2}\right)$ that the additive group generated by $\nu\left(\left\{t_{0}, t_{1}, \ldots, t_{k}\right\}\right)$ is discrete. Thus it is enough to apply Lemma 4 to $f_{t_{0}}, f_{t_{1}}, \ldots, f_{t_{k}}$.

Proof of Proposition 2. Each of statements (i) and (iii) implies that actually hypothesis $\left(\mathrm{H}_{2}\right)$ is satisfied. Thus they are equivalent on account of Lemma 4.

(ii) $\Rightarrow$ (iii). Assume that $L_{\mathcal{F}}=\{a, b\}$. Then Lemma 2 and [6, Theorem 2] yield a homeomorphism $h$ of $(a, b)$ onto itself and a homomorphism $n: G \rightarrow$ $\mathbb{Z}$ such that

$$
f_{t}=h^{n(\nu(t))}, \quad t \in T .
$$

Clearly $h$ has no fixed points, so it is enough to put $m=n \circ \nu$.

(iii) $\Rightarrow$ (ii). Assume (iii). Then $L_{\mathcal{F}}$ is simply the set of all cluster points of $\left\{h^{n}\left(x_{0}\right): n \in \mathbb{Z}\right\}$, where $x_{0}$ is an arbitrary point of $(a, b)$. Thus, since $h$ has no fixed points, $L_{\mathcal{F}}=\{a, b\}$.

Lemma 5. Let $f, g \in \mathcal{A}$ commute and let $\alpha:(a, b) \rightarrow \mathbb{R}$ be a monotonic solution of the system

$$
\alpha(f(x))=\alpha(x)+1, \quad \alpha(g(x))=\alpha(x)+c
$$

for some $c \in \mathbb{R}$. Then $c=\nu(f, g)$.

Proof. Without loss of generality we may assume that $f<\mathrm{Id}$ and $\alpha(x)=0$ for an $x \in(a, b)$. Then it follows from (10) that $\alpha$ is decreasing

$\left(^{2}\right)$ In fact it is enough to assume here that $f_{t}, t \in T$, are elements of $\mathcal{A}$. 
and

$$
\alpha\left(f^{n}(x)\right)=\alpha(x)+n \quad \text { and } \quad \alpha\left(g^{n}(x)\right)=\alpha(x)+n c, \quad n \in \mathbb{Z} .
$$

Let $\left(s_{k}(x): k \in \mathbb{N}\right)$ be a sequence of integers satisfying (2). Then

$$
\alpha\left(f^{s_{k}(x)}(x)\right) \leq \alpha\left(g^{k}(x)\right) \leq \alpha\left(f^{s_{k}(x)+1}(x)\right), \quad k \in \mathbb{N},
$$

whence, by (11), $s_{k}(x) \leq k c \leq s_{k}(x)+1, k \in \mathbb{N}$, and, consequently, $\nu(f, g)=c$.

Proof of Theorem 1. (i) By Proposition 2 there exist a homeomorphism $h \in \mathcal{A}$ and a function $m: T \rightarrow \mathbb{Z}$ satisfying (3). According to (3) we have $m(t) \neq 0, t \in T$, and (cf. Remarks 5(i) and 2(ii))

$$
\nu(t)=m(t) / m\left(t_{0}\right), \quad t \in T .
$$

Notice also that $h$, being an element of $\mathcal{A}$, is a strictly increasing function.

It follows from [3, Theorem 2.1 and Lemma 5.1] that the equation

$$
\alpha(h(x))=\alpha(x)+1 / m\left(t_{0}\right)
$$

has a homeomorphic solution depending on an arbitrary function. To complete the proof in this case it is enough to observe that if $\alpha:(a, b) \rightarrow \mathbb{R}$ is a solution of (13), then, by (3) and (12),

$$
\alpha\left(f_{t}(x)\right)=\alpha\left(h^{m(t)}(x)\right)=\alpha(x)+m(t) / m\left(t_{0}\right)=\alpha(x)+\nu(t)
$$

for every $x \in(a, b)$ and $t \in T$, i.e. $\alpha$ satisfies (A).

(ii) Let $\gamma$ be any homeomorphism of $(a, b)$ onto $\mathbb{R}$ satisfying (cf. [3, Theorem 2.1 and Lemma 5.1]) the equation

$$
\gamma\left(f_{t_{0}}(x)\right)=\gamma(x)+1 \text {. }
$$

Putting

$$
g_{t}=\gamma \circ f_{t} \circ \gamma^{-1}, \quad t \in T
$$

we obtain pairwise commuting homeomorphisms of $\mathbb{R}$ onto itself having no fixed points. Moreover,

$$
g_{t_{0}}(u)=u+1, \quad u \in \mathbb{R},
$$

the functions $g_{t}, t \in T$, satisfy hypothesis $\left(\mathrm{H}_{1}\right)$ with $a=-\infty$ and $b=\infty$, and

$$
\nu\left(g_{t_{0}}, g_{t}\right)=\nu\left(f_{t_{0}}, f_{t}\right)=\nu(t), \quad t \in T .
$$

In particular, since $g_{t} \circ g_{t_{0}}=g_{t_{0}} \circ g_{t}$, we have

$$
g_{t}(u+1)=g_{t}(u)+1, \quad u \in \mathbb{R}, t \in T .
$$

Denote by $S^{1}$ the unit circle $\{z \in \mathbb{C}:|z|=1\}$ with positive orientation. If $z_{1}, z_{2} \in S^{1}$, then there are (unique) reals $u_{1}, u_{2}$ such that $0 \leq u_{1} \leq u_{2}<$ 
$u_{1}+1<2$ and $z_{1}=e^{2 \pi i u_{1}}, z_{2}=e^{2 \pi i u_{2}}$, so we can correctly define the arc $\overrightarrow{\left[z_{1}, z_{2}\right)}$ putting

$$
\overrightarrow{\left[z_{1}, z_{2}\right)}=\left\{e^{2 \pi i u}: u \in\left[u_{1}, u_{2}\right)\right\}
$$

Set

$$
h_{t}=g_{t}-\left[g_{t}(0)\right], \quad t \in T,
$$

where $[x]$ stands for the greatest integer not exceeding $x$. It follows from (15) that

$$
h_{t}(u+1)=h_{t}(u)+1, \quad u \in \mathbb{R}, t \in T .
$$

Thus the formula

$$
H_{t}\left(e^{2 \pi i u}\right)=e^{2 \pi i h_{t}(u)}
$$

correctly defines homeomorphisms $H_{t}, t \in T$, of $S^{1}$ onto itself. Since, by $(15)$,

$$
g_{t}(u+p)=g_{t}(u)+p, \quad u \in \mathbb{R}, t \in T, p \in \mathbb{Z},
$$

it follows from (16) that $h_{t}, t \in T$, are pairwise commuting. Consequently, so are $H_{t}, t \in T$.

Fix a $z_{0} \in S^{1}$. We shall prove that the orbit

$$
\left\{H_{t_{1}}^{n_{1}} \circ \ldots \circ H_{t_{k}}^{n_{k}}\left(z_{0}\right): k \in \mathbb{N}, t_{1}, \ldots, t_{k} \in T, n_{1}, \ldots, n_{k} \in \mathbb{Z}\right\}
$$

is infinite. Let $z_{0}=e^{2 \pi i u_{0}}$ for a $u_{0} \in \mathbb{R}$. Fix a $p \in \mathbb{N}$. By the density of $G$ we can find $k \in \mathbb{N}, t_{1}, \ldots, t_{k} \in T$ and $n_{1}, \ldots, n_{k} \in \mathbb{Z}$ such that

$$
0<n_{1} \nu\left(t_{1}\right)+\ldots+n_{k} \nu\left(t_{k}\right)<1 / p .
$$

Suppose that

$$
H_{t_{1}}^{j n_{1}} \circ \ldots \circ H_{t_{k}}^{j n_{k}}\left(z_{0}\right)=H_{t_{1}}^{l n_{1}} \circ \ldots \circ H_{t_{k}}^{l n_{k}}\left(z_{0}\right)
$$

for some $j, l \in\{0, \ldots, p\}$. Then

$$
g_{t_{1}}^{j n_{1}} \circ \ldots \circ g_{t_{k}}^{j n_{k}}\left(u_{0}\right)=g_{t_{1}}^{l n_{1}} \circ \ldots \circ g_{t_{k}}^{l n_{k}}\left(u_{0}\right)+m
$$

for an $m \in \mathbb{Z}$, i.e.

$$
g_{t_{1}}^{j n_{1}} \circ \ldots \circ g_{t_{k}}^{j n_{k}}\left(u_{0}\right)=g_{t_{0}}^{m} \circ g_{t_{1}}^{l n_{1}} \circ \ldots \circ g_{t_{k}}^{l n_{k}}\left(u_{0}\right),
$$

whence, according to [2, Lemma 4.14],

$$
(j-l)\left(n_{1} \nu\left(t_{1}\right)+\ldots+n_{k} \nu\left(t_{k}\right)\right)=m \nu\left(t_{0}\right)=m .
$$

Thus by (20) we obtain $j=l$. In this way we have proved that for each $p \in \mathbb{N}$ the orbit (19) has at least $p$ different points, which means that it is infinite.

Theorem 2 yields a Borel probability measure $\mu$ on $S^{1}$ such that

$$
\mu\left(H_{t}(A)\right)=\mu(A), \quad t \in T,
$$


for every Borel subset $A$ of $S^{1}$. Define $\beta_{0}:[0,1) \rightarrow \mathbb{R}$ and $\beta: \mathbb{R} \rightarrow \mathbb{R}$ by

$$
\beta_{0}(u)=\mu\left(\overrightarrow{\left[1, e^{2 \pi i u}\right)}\right) \text { and } \beta(u)=\beta_{0}(u-[u])+[u] .
$$

Since $\beta$ is the distribution of a measure, it is increasing and left continuous. Fix a $u_{0} \in \mathbb{R}$ and put $z_{0}=e^{2 \pi i u_{0}}$ and $c=\beta\left(u_{0^{+}}\right)-\beta\left(u_{0}\right)$. Then $\mu\left(\left\{z_{0}\right\}\right)=c$ and (21) implies that

$$
\mu\left(\left\{H_{t_{1}}^{n_{1}} \circ \ldots \circ H_{t_{k}}^{n_{k}}\left(z_{0}\right)\right\}\right)=\mu\left(\left\{z_{0}\right\}\right)=c
$$

for any $k \in \mathbb{N}, t_{1}, \ldots, t_{k} \in T$ and $n_{1}, \ldots, n_{k} \in \mathbb{Z}$. Thus, since the orbit (19) is infinite, we have $c=0$. This means that $\beta$ is continuous.

We shall show that

$$
\beta\left(g_{t}(u)\right)=\beta(u)+\nu(t), \quad t \in T .
$$

By the definition of $\beta$ we have

$$
\beta(u+1)=\beta(u)+1, \quad u \in \mathbb{R} .
$$

Fix a $t \in T$. It follows from $\left(\mathrm{H}_{1}\right)$ that either there is a $p \in \mathbb{Z}$ for which

$$
g_{t}(u)=g_{t_{0}}^{p}(u), \quad u \in \mathbb{R},
$$

or for each $p \in \mathbb{Z}$,

$$
g_{t}(u) \neq g_{t_{0}}^{p}(u), \quad u \in \mathbb{R} .
$$

In the first case we have $g_{t}(u)=u+p, u \in \mathbb{R}$, so, using (23), we see that for any $u \in \mathbb{R}$,

$$
\beta\left(g_{t}(u)\right)=\beta(u+p)=\beta(u)+p=\beta(u)+\nu(t) .
$$

Thus we can assume that $g_{t}(u) \neq u+p, u \in \mathbb{R}, p \in \mathbb{Z}$. Then (cf. (16))

$$
u<h_{t}(u)<u+1, \quad u \in \mathbb{R} .
$$

Put $u_{0}=h_{t}^{-1}(1)$ and observe that $u_{0} \in(0,1)$ by $(24)$. We now verify that

$$
H_{t}\left(e^{2 \pi i u}\right) \in \overrightarrow{\left[H_{t}(1), 1\right)}, \quad u \in\left[0, u_{0}\right),
$$

and

$$
H_{t}\left(e^{2 \pi i u}\right) \in \overrightarrow{\left[1, H_{t}(1)\right)}, \quad u \in\left[u_{0}, 1\right) .
$$

Indeed, if $u \in\left[0, u_{0}\right)$, then, by $(24), h_{t}(0) \leq h_{t}(u)<h_{t}\left(u_{0}\right)=1<h_{t}(0)+1$, whence

$$
H_{t}\left(e^{2 \pi i u}\right)=e^{2 \pi i h_{t}(u)} \in \overrightarrow{\left[e^{2 \pi i h_{t}(0)}, e^{2 \pi i}\right)}=\overrightarrow{\left[H_{t}(1), 1\right)} .
$$

Similarly, if $u \in\left[u_{0}, 1\right)$, then, by (24) and (17), $0 \leq h_{t}(u)-1<h_{t}(1)-1=$ $h_{t}(0)$, whence

$$
H_{t}\left(e^{2 \pi i u}\right)=e^{2 \pi i\left(h_{t}(u)-1\right)} \in \overrightarrow{\left[e^{0}, e^{2 \pi i h_{t}(0)}\right)}=\overrightarrow{\left[1, H_{t}(1)\right)} .
$$


Let $c_{t}=\mu\left(\overrightarrow{\left[1, H_{t}(1)\right)}\right)$. Fix a $u \in\left[0, u_{0}\right)$ and put $z=e^{2 \pi i u}$. Then $0<h_{t}(u)<1$ and, by $(25), H_{t}(z) \in \overrightarrow{\left[H_{t}(1), 1\right)}$. Thus $(21)$ implies that

$$
\begin{aligned}
\beta\left(h_{t}(u)\right) & =\beta_{0}\left(h_{t}(u)\right)=\mu\left(\overrightarrow{\left(1, e^{2 \pi i h_{t}(u)}\right)}\right)=\mu\left(\overrightarrow{\left[1, H_{t}(z)\right)}\right) \\
& =\mu\left(\overrightarrow{\left(1, H_{t}(1)\right)}\right)+\mu\left(\overrightarrow{\left[H_{t}(1), H_{t}(z)\right)}\right)=c_{t}+\mu\left(H_{t}(\overrightarrow{[1, z)})\right) \\
& =c_{t}+\mu(\overrightarrow{[1, z)})=c_{t}+\beta_{0}(u)=c_{t}+\beta(u) .
\end{aligned}
$$

Now fix a $u \in\left[u_{0}, 1\right)$ and let $z=e^{2 \pi i u}$. Then $1 \leq h_{t}(u)<2$ and it follows from $(26)$ that $H_{t}(z) \in \overrightarrow{\left[1, H_{t}(1)\right)}$. Therefore, in view of $(21)$,

$$
\begin{aligned}
\beta\left(h_{t}(u)\right) & =\beta_{0}\left(h_{t}(u)-1\right)+1=1+\mu\left(\overrightarrow{\left(1, e^{2 \pi i\left(h_{t}(u)-1\right)}\right)}\right) \\
& =1+\mu\left(\overrightarrow{\left[1, e^{2 \pi i h_{t}(u)}\right)}\right)=1+\mu\left(\overrightarrow{\left[1, H_{t}(z)\right)}\right) \\
& =1+\mu\left(\overrightarrow{\left[1, H_{t}(1)\right)}\right)-\mu\left(\overrightarrow{\left[H_{t}(z), H_{t}(1)\right)}\right) \\
& =1+c_{t}-\mu\left(H_{t}(\overrightarrow{[z, 1)})\right)=1+c_{t}-\mu(\overrightarrow{[z, 1)}) \\
& =c_{t}+\mu(\overrightarrow{[1, z)})=c_{t}+\beta_{0}(u)=c_{t}+\beta(u) .
\end{aligned}
$$

Therefore, if $u \in[0,1)$, then, by (16) and (23),

$$
\beta\left(g_{t}(u)\right)=\beta\left(h_{t}(u)+\left[g_{t}(0)\right]\right)=\beta\left(h_{t}(u)\right)+\left[g_{t}(0)\right]=\beta(u)+c_{t}+\left[g_{t}(0)\right] .
$$

Hence and from (18) and (23) it follows that for any $u \in \mathbb{R}$,

$$
\begin{aligned}
\beta\left(g_{t}(u)\right) & =\beta\left(g_{t}(u-[u])\right)+[u]=\beta(u-[u])+c_{t}+\left[g_{t}(0)\right]+[u] \\
& =\beta(u)+c_{t}+\left[g_{t}(0)\right] .
\end{aligned}
$$

Using Lemma 5 we infer that actually $c_{t}+\left[g_{t}(0)\right]=\nu(t)$. Consequently,

$$
\beta\left(g_{t}(u)\right)=\beta(u)+\nu(t), \quad u \in \mathbb{R} .
$$

Put $\alpha=\beta \circ \gamma$. Then $\alpha$ is continuous and monotonic. Moreover, by (14) and (22), we have for any $t \in T$ and $x \in(a, b)$,

$$
\begin{aligned}
\alpha\left(f_{t}(x)\right) & =\beta\left(\gamma\left(f_{t}(x)\right)\right)=\beta\left(g_{t}(\gamma(x))\right) \\
& =\beta(\gamma(x))+\nu(t)=\alpha(x)+\nu(t),
\end{aligned}
$$

that is, $\alpha$ is a solution of $(\mathrm{A})$.

It follows from Propositions 1 and 2 that $L_{\mathcal{F}} \cap(a, b) \neq \emptyset$. Let $\alpha_{1}, \alpha_{2}$ : $(a, b) \rightarrow \mathbb{R}$ be solutions of $(\mathrm{A})$ which are continuous at a common point $x_{0} \in L_{\mathcal{F}} \cap(a, b)$. Put $\alpha=\alpha_{2}-\alpha_{1}$. If $x \in(a, b)$, then $\alpha\left(f_{t}(x)\right)=\alpha(x), t \in T$, which means that $\left.\alpha\right|_{C_{\mathcal{F}}(x)}$ is constant. Thus, by the continuity of $\alpha$ at $x_{0}$,

$$
\alpha(x)=\alpha\left(x_{0}\right), \quad x \in(a, b),
$$

and, consequently, $\alpha_{2}=\alpha_{1}+c$ for a $c \in \mathbb{R}$. 
It is clear that $L_{\mathcal{F}} \subset \operatorname{cl} C_{\mathcal{F}}(x), x \in(a, b)$. If $x \in L_{\mathcal{F}} \cap(a, b)$, then, by Proposition 1(ii), $C_{\mathcal{F}}(x) \subset L_{\mathcal{F}}$. Consequently,

$$
\operatorname{cl} C_{\mathcal{F}}(x)=L_{\mathcal{F}}, \quad x \in L_{\mathcal{F}} \cap(a, b) .
$$

Let $\alpha:(a, b) \rightarrow \mathbb{R}$ be a continuous solution of $(\mathrm{A})$. Then $\alpha$ is monotonic and

$$
\alpha\left(C_{\mathcal{F}}(x)\right)=\alpha(x)+G, \quad x \in(a, b) .
$$

Fix an $x \in L_{\mathcal{F}} \cap(a, b)$. Since $\alpha$ is continuous and monotonic and $G$ is dense in $\mathbb{R}$, from (28) we have

$$
\begin{aligned}
\alpha\left(\operatorname{cl} C_{\mathcal{F}}(x) \cap(a, b)\right) & =\operatorname{cl} \alpha\left(C_{\mathcal{F}}(x)\right)=\operatorname{cl}(\alpha(x)+G) \\
& =\alpha(x)+\operatorname{cl} G=\mathbb{R},
\end{aligned}
$$

which means (cf. (27)) that

$$
\alpha\left(L_{\mathcal{F}} \cap(a, b)\right)=\mathbb{R} .
$$

Now assume that $\alpha$ is invertible. Then it is a homeomorphism and, by $(29), L_{\mathcal{F}}$ is an interval. Consequently, Proposition 1(iii) shows that $L_{\mathcal{F}}=[a, b]$.

Conversely, assume that $L_{\mathcal{F}}=[a, b]$ and suppose that $\alpha$ is not invertible. Let $I$ be a proper interval of constancy of $\alpha$ and let $x_{0} \in(a, b)$. Since $I \subset L_{\mathcal{F}}$, we can find $u, v \in I \cap C_{\mathcal{F}}\left(x_{0}\right)$ such that $u \neq v$. Choose $k \in \mathbb{N}, t_{1}, \ldots, t_{k} \in T$ and $n_{1}, \ldots, n_{k}, m_{1}, \ldots, m_{k} \in \mathbb{Z}$ for which

$$
u=f_{t_{1}}^{n_{1}} \circ \ldots \circ f_{t_{k}}^{n_{k}}\left(x_{0}\right) \text { and } v=f_{t_{1}}^{m_{1}} \circ \ldots \circ f_{t_{k}}^{m_{k}}\left(x_{0}\right) .
$$

Then, by $(\mathrm{A})$,

$$
\begin{aligned}
\alpha\left(x_{0}\right)+ & n_{1} \nu\left(t_{1}\right)+\ldots+n_{k} \nu\left(t_{k}\right) \\
& =\alpha\left(f_{t_{1}}^{n_{1}} \circ \ldots \circ f_{t_{k}}^{n_{k}}\left(x_{0}\right)\right)=\alpha(u)=\alpha(v) \\
& =\alpha\left(f_{t_{1}}^{m_{1}} \circ \ldots \circ f_{t_{k}}^{m_{k}}\left(x_{0}\right)\right)=\alpha\left(x_{0}\right)+m_{1} \nu\left(t_{1}\right)+\ldots+m_{k} \nu\left(t_{k}\right),
\end{aligned}
$$

whence $n_{1} \nu\left(t_{1}\right)+\ldots+n_{k} \nu\left(t_{k}\right)=m_{1} \nu\left(t_{1}\right)+\ldots+m_{k} \nu\left(t_{k}\right)$. Now Lemma 1 yields

$$
u=f_{t_{1}}^{n_{1}} \circ \ldots \circ f_{t_{k}}^{n_{k}}\left(x_{0}\right)=f_{t_{1}}^{m_{1}} \circ \ldots \circ f_{t_{k}}^{m_{k}}\left(x_{0}\right)=v,
$$

a contradiction. Thus $\alpha$ is invertible, which completes the proof.

3. Some consequences. Notice that proving the "uniqueness" part of Theorem 1 for $G$ dense in $\mathbb{R}$, we have actually proved the uniqueness of solutions in the class of functions continuous at a point of $L_{\mathcal{F}} \cap(a, b)$. Thus we also have the following fact.

COROLlary 1. Let $\mathcal{F}=\left\{f_{t}: t \in T\right\}$ satisfy hypothesis $\left(\mathrm{H}_{1}\right)$ and assume that $G$ is dense in $\mathbb{R}$. If $\alpha:(a, b) \rightarrow \mathbb{R}$ is a solution of system $(\mathrm{A})$ which is continuous at a point of $L_{\mathcal{F}} \cap(a, b)$, then $\alpha$ is continuous. 
By Remark 5(ii) the following result can be immediately deduced from Theorem 1.

THEOREM 3. Let $\mathcal{F}=\left\{f_{t}: t \in T\right\}$ satisfy hypothesis $\left(\mathrm{H}_{2}\right)$.

(i) Assume that the group $G$ is discrete. Then system (A) has a homeomorphic solution depending on an arbitrary function.

(ii) Assume that $G$ is dense in $\mathbb{R}$. Then (A) has a unique (up to an additive constant) continuous solution $\alpha:(a, b) \rightarrow \mathbb{R}$. This solution is monotonic and $\alpha\left(L_{\mathcal{F}} \cap(a, b)\right)=\mathbb{R}$. Moreover, $\alpha$ is invertible if and only if $L_{\mathcal{F}}=[a, b]$.

Corollary 2. Let $\mathcal{F}=\left\{f_{t}: t \in \mathbb{Q}\right\} \subseteq \mathcal{A}$ satisfy $\left({ }^{3}\right)$

$$
f_{s} \circ f_{t}=f_{s+t}, \quad s, t \in \mathbb{Q} \text {. }
$$

Then the system

$$
\alpha\left(f_{t}(x)\right)=\alpha(x)+t, \quad t \in \mathbb{Q},
$$

has a unique (up to an additive constant) continuous solution $\alpha:(a, b) \rightarrow \mathbb{R}$. This solution is monotonic and $\alpha\left(L_{\mathcal{F}} \cap(a, b)\right)=\mathbb{R}$. Moreover, $\alpha$ is invertible if and only if $L_{\mathcal{F}}=[a, b]$.

Proof. It follows from (30) that $f_{m / n}^{n}=f_{m}=f_{1}^{m}, m \in \mathbb{Z}, n \in \mathbb{N}$. Thus, putting $T=\mathbb{Q}$ and $t_{0}=1$, we see that $\mathcal{F}$ satisfies $\left(\mathrm{H}_{2}\right)$ and (cf. Remark 2(ii)) $\nu\left(f_{1}, f_{m / n}\right)=m / n, m \in \mathbb{Z}, n \in \mathbb{N}$, i.e. $\nu(t)=t, t \in T$. Hence $G=\mathbb{Q}$ and to get the assertion it is enough to apply Theorem 3(ii).

Before stating another consequence of Theorem 1 we need two more facts.

Lemma 6. Let $f, g, h$ be pairwise commuting elements of $\mathcal{A}$ and assume that the pair $(f, g)$ is aperiodic. Then any continuous solution $\alpha:(a, b) \rightarrow \mathbb{R}$ of the system

$$
\alpha(f(x))=\alpha(x)+1, \quad \alpha(g(x))=\alpha(x)+\nu(f, g)
$$

satisfies also the equation

$$
\alpha(h(x))=\alpha(x)+\nu(f, h) .
$$

Proof. Let $\alpha:(a, b) \rightarrow \mathbb{R}$ be a continuous solution of (31) and put $\beta=\alpha \circ h$. Then for any $x \in(a, b)$,

$$
\beta(f(x))=\alpha(h(f(x)))=\alpha(f(h(x)))=\alpha(h(x))+1=\beta(x)+1
$$

and

$$
\begin{aligned}
\beta(g(x)) & =\alpha(h(g(x)))=\alpha(g(h(x)))=\alpha(h(x))+\nu(f, g) \\
& =\beta(x)+\nu(f, g) .
\end{aligned}
$$

Notice that $\{f, g\}$ satisfies $\left(\mathrm{H}_{1}\right)$. Moreover, by Remark $2(\mathrm{i}), \nu(f, g)$ is irrational, so the additive group generated by $\{1, \nu(f, g)\}$ is dense in $\mathbb{R}$.

$\left({ }^{3}\right)$ Such a family is said to be a rational flow or rational iteration group. 
Thus, by Theorem 1 (ii), $\alpha$ is monotonic and $\beta=\alpha+c$ for a $c \in \mathbb{R}$, which means that $\alpha(h(x))=\alpha(x)+c, x \in(a, b)$. Now the conclusion follows from Lemma 5.

Remark 6. Let $f, g, h$ be pairwise commuting elements of $\mathcal{A}$ and assume that $(f, g)$ and $(f, h)$ are aperiodic. Then $L_{\{f, g\}}=L_{\{f, h\}}$.

Proof. It is enough to prove that $L_{\{f, h\}} \subset L_{\{f, g\}}$. By Theorem 1(ii) applied to $\{f, g\}$ there exists a continuous monotonic solution $\alpha:(a, b) \rightarrow \mathbb{R}$ of (31) satisfying

$$
\alpha\left(L_{\{f, g\}} \cap(a, b)\right)=\mathbb{R} .
$$

In view of Proposition 1(iii), $L_{\{f, g\}}$ is closed and $\{a, b\} \subset L_{\{f, g\}}$. Thus there are pairwise disjoint open intervals $I_{n}, n \in \mathbb{N}$, such that

$$
[a, b] \backslash L_{\{f, g\}}=\bigcup_{n=1}^{\infty} I_{n} .
$$

Suppose that $L_{\{f, h\}} \not \subset L_{\{f, g\}}$ and let $x \in L_{\{f, h\}} \backslash L_{\{f, g\}}$. Choose a $k \in \mathbb{N}$ with $x \in L_{\{f, h\}} \cap I_{k}$. Then (cf. Proposition 1(i)) there exist $u, v \in$ $C_{\{f, h\}}(x) \cap I_{k}, u \neq v$. Let $n, m, p, q \in \mathbb{Z}$ be such that $u=f^{n} \circ h^{m}(x)$ and $v=f^{p} \circ h^{q}(x)$. It follows from (32) and the monotonicity of $\alpha$ that $\left.\alpha\right|_{I_{k}}$ is constant. Therefore, by Lemma 6 ,

$$
\begin{aligned}
\alpha(x)+n+m \nu(f, h) & =\alpha\left(f^{n} \circ h^{m}(x)\right)=\alpha(u)=\alpha(v) \\
& =\alpha\left(f^{p} \circ h^{q}(x)\right)=\alpha(x)+p+q \nu(f, h),
\end{aligned}
$$

whence, by irrationality of $\nu(f, h)$ (cf. Remark 2(i)), $n=p$ and $m=q$. Consequently, $u=v$, a contradiction.

In Theorem 4 below $L$ denotes the set $L_{\left\{f_{t_{0}}, f_{t}\right\}}$, where $\left(f_{t_{0}}, f_{t}\right)$ is an arbitrary aperiodic pair. Hypothesis $\left(\mathrm{H}_{3}\right)$ ensures the existence of such a pair and Remark 6 shows that $L$ does not depend on the choice of it.

Theorem 4. Assume $\left(\mathrm{H}_{3}\right)$. Then system (A) has a unique (up to an additive constant) continuous solution $\alpha:(a, b) \rightarrow \mathbb{R}$. This solution is monotonic and $\alpha(L \cap(a, b))=\mathbb{R}$. Moreover, $\alpha$ is invertible if and only if $L=[a, b]$.

Proof. Let $t_{1} \in T$ be such that $\left(f_{t_{0}}, f_{t_{1}}\right)$ is aperiodic and

$$
f_{t_{0}} \circ f_{t}=f_{t} \circ f_{t_{0}} \quad \text { and } f_{t_{1}} \circ f_{t}=f_{t} \circ f_{t_{1}}, \quad t \in T \text {. }
$$

By Remark 5(iii), $\left\{f_{t_{0}}, f_{t_{1}}\right\}$ satisfies $\left(\mathrm{H}_{1}\right)$. Moreover, it follows from Remark 2(i) that $\nu\left(t_{1}\right)$ is irrational. Since $\nu\left(t_{0}\right)=1$, this means that the additive group generated by $\left\{\nu\left(t_{0}\right), \nu\left(t_{1}\right)\right\}$ is dense in $\mathbb{R}$. Therefore, according to Theorem 1(ii), there exists a unique (up to an additive constant) continuous solution $\alpha:(a, b) \rightarrow \mathbb{R}$ of the system

$\left(\mathrm{A}_{t_{1}}\right) \quad \alpha\left(f_{t_{0}}(x)\right)=\alpha(x)+1, \quad \alpha\left(f_{t_{1}}(x)\right)=\alpha(x)+\nu\left(t_{1}\right)$. 
Lemma 6 shows that $\alpha$ satisfies (A). By Theorem 1(ii), $\alpha$ has all the remaining desired properties.

Notice that we have just proved also the following fact.

Rem ark 7. Assume $\left(\mathrm{H}_{3}\right)$. If $t_{1} \in T$ is such that $\left(f_{t_{0}}, f_{t_{1}}\right)$ is aperiodic and $(33)$ is satisfied, then systems $(A)$ and $\left(A_{t_{1}}\right)$ have the same sets of continuous solutions.

Finally, we return to system (1). It turns out that in some cases, (1) can be reduced to (A). First notice that the following fact follows immediately from Lemma 5.

Corollary 3. Let $f_{t}, t \in T$, be pairwise commuting elements of $\mathcal{A}$ and suppose $\lambda: T \rightarrow \mathbb{R}$ does not vanish at $t_{0}$. If system (1) has a monotonic solution defined on $(a, b)$, then $\lambda=c \nu$ for a non-zero $c \in \mathbb{R}$.

Regarding continuous solutions of (1) we can prove the following.

Corollary 4. Let $\left(\mathrm{H}_{2}\right)$ or $\left(\mathrm{H}_{3}\right)$ be satisfied and suppose $\lambda: T \rightarrow \mathbb{R}$ does not vanish at $t_{0}$. Then (1) has a continuous solution defined on $(a, b)$ if and only if $\lambda=c \nu$ for a non-zero $c \in \mathbb{R}$.

Proof. Assume, for instance, that $f_{t_{0}}>$ Id and let $\alpha:(a, b) \rightarrow \mathbb{R}$ be a continuous solution of (1). Then $\alpha_{1}=\alpha / \lambda\left(t_{0}\right)$ satisfies

$$
\alpha_{1}\left(f_{t}(x)\right)=\alpha_{1}(x)+\lambda(t) / \lambda\left(t_{0}\right), \quad x \in(a, b), t \in T .
$$

Fix a continuous solution $\alpha_{2}:(a, b) \rightarrow \mathbb{R}$ of $(\mathrm{A})$ (cf. Theorems 3 and 4 ) and put $\alpha_{0}=\alpha_{1}-\alpha_{2}$. By (34) and (A) we have for any $t \in T$ and $x \in(a, b)$,

$$
\begin{aligned}
\alpha_{0}\left(f_{t}(x)\right) & =\alpha_{1}\left(f_{t}(x)\right)-\alpha_{2}\left(f_{t}(x)\right) \\
& =\left(\alpha_{1}(x)+\lambda(t) / \lambda\left(t_{0}\right)\right)-\left(\alpha_{2}(x)+\nu(t)\right) \\
& =\alpha_{0}(x)+\lambda(t) / \lambda\left(t_{0}\right)-\nu(t) .
\end{aligned}
$$

In particular,

$$
\alpha_{0}\left(f_{t_{0}}(x)\right)=\alpha_{0}(x), \quad x \in(a, b) .
$$

Therefore $\alpha_{0}((a, b))=\alpha_{0}\left(\left[x_{0}, f_{t_{0}}\left(x_{0}\right)\right]\right)$, where $x_{0}$ is an arbitrary point of $(a, b)$. Hence, $\alpha_{0}$ is bounded. Moreover,

$$
\alpha_{2}\left(f_{t}^{n}(x)\right)=\alpha_{0}(x)+n\left(\lambda(t) / \lambda\left(t_{0}\right)-\nu(t)\right), \quad x \in(a, b), t \in T,
$$

for every $n \in \mathbb{N}$. Therefore the boundedness of $\alpha_{0}$ implies that $\lambda(t) / \lambda\left(t_{0}\right)-$ $\nu(t)=0, t \in T$, i.e. $\lambda=\lambda\left(t_{0}\right) \nu$. The converse statement follows from Theorems 3 and 4 .

Acknowledgements. The research of the first author was supported by the Mathematics Department of Silesian University (Iterative Functional Equations and Real Analysis program). 


\section{References}

[1] J. Dugundji and A. Granas, Fixed Point Theory, Vol. 1, Monografie Mat. 61, Polish Scientific Publishers, Warszawa, 1982.

[2] W. Jarczyk, A recurrent method of solving iterative functional equations, Prace Naukowe Uniw. Śląsk. Katowic. 1206, Katowice, 1991.

[3] M. Kuczma, Functional Equations in a Single Variable, Monografie Mat. 46, Polish Scientific Publishers, Warszawa, 1968.

[4] M. C. Zd un, Note on commutable functions, Aequationes Math. 36 (1988), 153-164.

[5] -, On simultaneous Abel equations, ibid. 38 (1989), 163-177.

[6] - On the orbits of disjoint groups of continuous functions, Rad. Mat., to appear.

[7] - Some remarks on the iterates of commuting functions, in: European Conference on Iteration Theory, Lisbon, 1991, J. P. Lampreia et al. (eds.), World Scientific, Singapore, 1992, 336-342.

INSTITUTE OF MATHEMATICS

SILESIAN UNIVERSITY

BANKOWA 14

40-007 KATOWICE, POLAND

\section{INSTITUTE OF MATHEMATICS PEDAGOGICAL UNIVERSITY PODCHORAŻ̇YCH 2} 30-084 KRAKÓW, POLAND

Reçu par la Rédaction le 15.12.1992 